\title{
The meaning of cognitive dysfunction in bipolar disorder: a risk factor or a specific form of dementia?
}

\author{
Cássio Silveira de Jesús'1, Marlos Vasconcelos Rocha1 ${ }^{1}$ Ângela Miranda-Scippa1,2 \\ 1 Program of Mood and Anxiety Disorders (CETHA), University Hospital, Federal University of Bahia, Salvador, BA, Brazil. \\ 2 Postgraduate Program in Medicine and Health, Federal University of Bahia, Salvador, BA, Brazil. Department of Neurosciences and Mental Health, Medical School, Federal University of Bahia, \\ Salvador, BA, Brazil.
}

Received: 07/25/2018 - Accepted: 08/08/2018

DOI: 10.1590/0101-60830000000174

Jesús CS et al. / Arch Clin Psychiatry. 2018;45(5):135

\section{Dear Editor,}

Bipolar disorder $(\mathrm{BD})$ is a chronic pathology with a worldwide prevalence of $2.4 \%$ in its classic presentations ${ }^{1}$. Studies show that the depressive, mania/hypomania crises are neurotoxic and cognitive impairment is present both in the acute phases as well as in euthymia. However, bipolar type I patients have more cognitive changes than type II patients, most likely due to greater toxicity of manic episodes, with greater release of inflammatory cytokines, reduction of brainderived neurotrophic factor (BDNF), higher oxidative stress and, consequently, loss of neuroprotective mechanisms ${ }^{2}$. In this sense, more than the time of disease duration, the number of manic episodes seems to determine the exacerbation of cognitive decline, mainly in patients presenting psychotic symptoms ${ }^{2,3}$.

In $\mathrm{BD}$ patients, cognitive deficits are found in several domains, such as processing speed, visuospatial abilities, verbal memory and attention, with the last two being the most impaired ${ }^{3}$. Furthermore, evidence shows that these deficits are associated with total brain atrophy, enlargement of the lateral ventricles, decrease of the corpus callosum volume, and the reduction of the hippocampus, findings that are also found in neurodegenerative diseases such as dementia ${ }^{4,5}$. Besides that, according to the theory of neuroprogression in $\mathrm{BD}$ stage 4, there are loss of autonomy due to cognitive and functional impairment, associated with enlargement ventricle and/or white matter hyperintensities, increase of tumor necrosis factor $\alpha$ (TNF- $\alpha$ ), increase of nitrotyrosine 3 , decrease of BDNF, increase of interleukins 6,10 , and glutathione transferase ${ }^{4}$.

So, although several studies point to BD as a risk factor for dementia, we question whether the cognitive dysfunctions present in some of these patients are not enough to affirm that there also a "bipolar dementia", just like it's done in other pathologies such as Parkinson's disease ${ }^{6}$ and epilepsy ${ }^{7}$. In fact, Parkinson's disease, epilepsy and BD patients who develop cognitive dysfunction do not present the changes in the cerebrospinal fluid as are described in Alzheimer's disease, such as low concentrations of beta amyloid protein and high concentrations of total and phosphorylated tau protein ${ }^{5}$. In $\mathrm{BD}$, like in these diseases, there is cognitive impairment; the deficits emerge over time with the recurrence of crises and there is no evidence pointing to a related or altered specific substance, leading to neurodegeneration ${ }^{3}$.
Recognizing $\mathrm{BD}$ as a pathology that might cause a subtype of dementia is important to reinforce the need for adequate diagnosis and treatment, bearing in mind the loss of functionality of the patients who present cognitive deficits. Furthermore, we should remember that BD has a high comorbidity with metabolic syndrome, which may also contribute toward microangiopathy and vascular dementia scenarios, whose physiopathology is different from dementia due to neurodegeneration. Thus, we pose the following question: is $\mathrm{BD}$ only a risk factor for dementia or must be considerate a distinct clinical entity?

\section{Disclosure}

The authors report no conflicts of interest.

\section{References}

1. Merikangas KR, Akiskal HS, Angst J, Greenberg PE, Hirschfeld RMA, Petukhova M, et al. Lifetime and 12-month prevalence of bipolar spectrum disorder in the national comorbidity survey replication. Arch Gen Psychiatry. 2007;64(5):543-52.

2. Piccinni A, Marazziti D, Callari A, Franceschini C, Veltri A, Bartolommei $\mathrm{N}$, et al. Bipolar disorder and dementia: a close link. Clin Neuropsychiatry. 2015;12(2):27-36.

3. Cardoso T, Bauer IE, Meyer TD, Kapczinski F, Soares JC. Neuroprogression and cognitive functioning in bipolar disorder: a systematic review. Curr Psychiatry Rep. 2015;17(9).

4. Roda A, Chendo I, Kunz M. Biomarkers and staging of bipolar disorder: a systematic review. Trends Psychiatry Psychother. 2015;37(1):3-11.

5. Forlenza OV, Aprahamian I, Radanovic M, Talib LL, Camargo MZA, Stella F, et al. Cognitive impairment in late-life bipolar disorder is not associated with Alzheimer's disease pathological signature in the cerebrospinal fluid. Bipolar Disord. 2016;18(1):63-70.

6. Hanagasi HA, Tufekcioglu Z, Emre M. Dementia in Parkinson's disease. J Neurol Sci. 2017;374(2016):26-31.

7. Breuer LEM, Grevers E, Boon P, Bernas A, Bergmans JWM, Besseling $\mathrm{RMH}$, et al. Cognitive deterioration in adult epilepsy: clinical characteristics of "Accelerated Cognitive Ageing." Acta Neurol Scand. 2017;136(1):47-53. 